\title{
Structure électronique du nitrure de bore cubique dans l'approximation de la densité électronique locale
}

\author{
S. Matar, V. Gonnet et G. Demazeau \\ L.C.S.-C.N.R.S., Université Bordeaux 1, 351 cours de la Libération, F-33405 Talence Cedex \\ France
}

(Reçu le 23 jullet 1993, révisé le 22 septembre 1993, accepté le 20 octobre 1993)

\begin{abstract}
Résumé. - La structure électronique du nitrure de bore cubique est réexaminée par calculs autocohérents utilisant la méthode ASW. Nos résultats sont comparés avec ceux calculés de la littérature. Les valeurs du paramètre de mallle, de l'inverse de la compressibilité, de la largeur de la bande de valence et à une moindre échelle des gaps direct et indirect, sont en accord relatif avec les valeurs expérimentales. Des sphères vides (pseudo-atomes avec $Z=0$ ) introduites dans les sites interstitiels créent des états au bas de la bande de conduction. Elles devraient jouer un rôle en modifiant la structure de bandes lorsque des atomes interstitiels sont introduits.
\end{abstract}

\begin{abstract}
The electronic structure of cubic boron nitride is revisited using ASW self-consistent calculations. Our results are confronted with calculated data from the literature. The equilibrium lattice constant, the bulk modulus, the valence band width and to a lesser extent the band gaps are found to be within reasonable agreement with experiment. Empty spheres (pseudo-atoms with $Z=0$ ) introduced at the interstitial sites create states at the bottom of the conduction band. They should play a role in modifying the band structure when interstitial atoms are introduced.
\end{abstract}

\section{Introduction.}

Le nitrure de bore BN peut être consıdéré comme le premıer des composés III-V. Il existe sous trois formes allotropıques: graphite, wurtzite et blende. Cette dernière forme a été jusqu'ıci préparée dans des conditions de hautes pressions et hautes températures. Elle présente un intérêt important du fait de ses applicatıons potentıelles lıées à ses propriétés physicochimiques exceptionnelles [1].

Malgré l'existence dans la littérature scientıfique d'un certain nombre de travaux sur l'étude de la structure électronıque de BN et des composés « III-V » [2-5], une approche analytique du rôle de l espace interstitiel - important dans la structure de la blende - et des modifications de distributions de charges induites entre $\mathrm{B}$ et $\mathrm{N}$ n'a pas été suffisamment abordée.

L'objectif de notre travall est d'adopter une telle approche pour le binaire $B N$ dans sa structure cubique à l'arde de calculs auto-cohérents de sa structure électronıque. 


\section{La méthode de calcul et les approxımations de base.}

Les calculs ont été effectués par la méthode ASW (augmented spherlcal wave) [6] basée sur deux approximations principales.

i) Celle de la fonctıonnelle de la densité électronique (DFT) stipule que les propriétés de l'état fondamental sont connues si la densité électronique l'est (ceci sous-tend le cycle autocohérent du calcul) [7]. Les effets d'échange et corrélation sont pris en compte dans le cadre de l'approximation de la densité électronıque locale (LDA) basée sur celle d'un gaz unıforme d'électrons (cf. [8] pour une référence récente).

1i) Celle de la sphère atomique (ASA), selon laquelle la maille élémentaire du cristal est partagée en sphères qui se recouvrent afin que leur volume total solt égal à celuı de la marle $\left(\Sigma V_{1} \Leftrightarrow \Sigma\left(4 / 3 \pi r_{l}^{3}\right)=V_{\text {malle, }}, l=\right.$ indice de sphère $)$.

Dans le cadre de cette deuxième approximatıon une remarque peut être soulevée. Pour les structures compactes (notamment les métaux et les alliages de structures cubique à faces centrées ou hexagonale compacte, etc...) on peut s'attendre à un faible recouvrement des sphères atomiques. En revanche pour des structures moins compactes ex. les alliages métal/non-métal, les oxydes ou encore la structure blende, la condition ASA ne peut être respectée sans que le recouvrement des seules sphères atomiques ne soit trop important pour conserver un sens physıque aux résultats. La contınuité de la densité électronıque sur tout le cristal est alors assurée par l'introduction de sphères vides (ES) - pseudo-atomes $(Z=0)$ dans les sites interstitiels de la structure. Les résidus de charge d'un atome vorsin d'une ES qui s'y trouvent transférés seront pris en compte lors du bilan global de charges, comme il en sera explicité pour BN-c.

La fonction d'onde sphérique est centrée sur et identifiée avec chaque sphère atomique [6]. L'ASW représentant l'état propre recherché pour chaque nombre quantique $\ell$ est obtenue par un processus d'augmentation faisant intervenır des combinarsons lınéares de fonctions sphérıques de type Hankel (tête de la fonction d'onde, ou milieu de la bande) et Bessel (queue de la fonction d'onde ou extrêmité de la bande). Pour un état $\ell$ donné on se servira des énergies propres des fonctions de Hankel pour établir des comparaisons.

\section{Posıtionnement des sphères atomiques pour les calculs ASW.}

La structure adoptée par la variété de hautes pression et température de BN est celle de la blende décrite dans le groupe d'espace $\mathrm{F}_{4}^{-} 3 \mathrm{~m}\left(\mathrm{~T}_{\mathrm{d}}^{2}\right)$. Avec B en (4a): $0,0,0$ et $\mathrm{N}$ en $(4 \mathrm{c}): 1 / 4$, $1 / 4,1 / 4$, cet empilement laisse autant de positıons interstitielles non occupées : (4b) $1 / 2,1 / 2$, $1 / 2$ et (4d) $3 / 4,3 / 4,3 / 4$, elles le seront par les sphères vides respectives de $B$ et de $N$ dans I'ASA.

\section{Calculs ASW de BN-c.}

\section{1 CRITÈRES DE FIABILITÉ des CALCUls.}

4.1.1 Rayons des sphères (ASA). - Le confinement des atomes avec leurs cortèges électroniques dans un volume donné et le transfert d'électrons résultant de la liarson chimıque contractée entre eux lors de leur positionnement dans le réseau cristallın donnent lıeu à une pression électronıque à la surface de la sphère atomıque. Pour un calcul ayant convergé, le produit de cette pression électronique par le volume de la sphère est une énergıe qui doit être mınımale pour l'ensemble des sphères atomıques en même temps que l'énergıe varıatıonnelle totale. Cecı conduit à optımıser les rayons des sphères. Le rapport des rayons $r_{\mathrm{B}, \mathrm{N}} / r_{\left(\mathrm{ES}_{\mathrm{B}}, \mathrm{ES}_{\mathrm{V}}\right)}$ ansı optımısé était égal à 1 pour BN-c (supposant les rayons de toutes les sphères égaux). 
Aucune recherche ultérieure portant sur les modifications des rapports des rayons de $\mathrm{B}$ et $\mathrm{N}$ d'une part ou de leurs sphères vides respectives d'une part n'a été effectuée.

4.1.2 Nombre de points $\mathbf{k}$. - Les calculs sont effectués pour un nombre de points $\mathbf{k}$ dans la première zone de Brillouin jusqu'à convergence, i e. jusqu'à ce qu'il n'y ait plus de transfert de charge entre les différents constituants : $|\Delta Q|<Q_{\text {conv }}$ avec $\Delta Q$ différence entre les charges provenant du nouveau et de l'ancien cycle de calcul et $Q_{\text {conv }}$ différence de charges constituant le critère de convergence; ex. $\Delta Q=10^{-4}$, valeur retenue pour nos calculs. Cependant cette convergence est obtenue pour un nombre donné de points $\mathbf{k}$ et il faut augmenter ce nombre pour avorr une meilleure fiabilité. Dans le cas de BN-c 12 points $\mathbf{k}$ suivant chacune des trois directions de l'espace i.e. 182 points $\mathbf{k}$ ont été nécessaires pour arriver à une parfaite convergence; l'énergie varuationnelle totale ne varie alors que de moins de $1 \mathrm{mRyd}$ $(1 \mathrm{Ryd}=13,6 \mathrm{eV})$.

4.2 RECHERCHE DES PARAMÈTRES D'ÉQUILIBRE. - Des calculs ont été effectués pour 4 paramètres autour de la valeur expérmentale de 7,002 u.a. (u.a. = unité atomique, 1 u.a. $=0,529 \AA$ ) afin de trouver la valeur d'équilibre du paramètre. Un ajustement quadratıque effectué pour ces quatre couples de valeurs $V_{l}, E(V A R)_{\imath}$ a permis d'obtenir d'une part le paramètre à l'équilibre correspondant à un minimum de l'énergie variationnelle : $a_{\text {éq }}=6.923$ u.a. (soit près de $2 \%$ inférieur à la valeur expérımentale), d'autre part Bo: module d'incompressibilıté de volume $=353 \mathrm{GPa}$. Cette dernière, égale à la valeur obtenue par Park et al. par calculs FLAPW [3], est inférieure à la valeur expérımentale : $456 \mathrm{GPa}$ (cf. [1]). Ceci pourrait être dû à l'utilisation de la DFT/LDA, où des grandeurs telles que $a_{\text {éq }}$ ou la compressibilité dérivées de l'énergie totale sont sous-estrmées.

L'ensemble de ces résultats permet de conférer une certaıne fiabilıté aux calculs de manıère à analyser dans une deuxième étape la structure électronique.

\subsection{RÉSUltats DES CALCUlS DE STRUCTURE ÉlECTRONIQUE.}

4.3.1 Charges partielles et énergies des états. - Pour nos calculs nous avons adopté comme base explicite $: \ell=0,1$ (états $\mathrm{s}$ et $\mathrm{p}$ ) et $\ell_{\max }=2$ pour $\mathrm{B}, \mathrm{N}$ et leurs sphères vides. Les états associés avec $\ell_{\max }$ reçoıvent les résıdus de charges correspondant à tous les états nonexplicitement considérés comme états de valence. Ceci provient du fait que la base des ondes sphériques est lımitée. La condition que les résıdus de charge soient systématiquement inférieurs aux occupations des états explicites constıtue un critère de convergence supplémentaire.

Les charges partıelles dans chaque sphère, pour les différents états relatıfs aux valeurs de $\ell$ sont données au tableau I (partie de gauche). Pour chaque type de sphère l'état $P_{\max }$ est caractérisé par une charge inférieure aux valeurs correspondant aux états $s$ et $p$. Des transferts de charges sélectıfs s'opèrent depuis les sphères atomiques de $\mathrm{B}$ et de $\mathrm{N}$ vers les $\mathrm{ES}$ en ce sens que les deux sphères vides ne sont pas équivalentes d'où leur désignation suivant chaque atome. En effet 1 l n'est pas possible d'aboutir au nombre d'électrons de valence (B : 3 ; $\mathrm{N}: 5 \Rightarrow Q$ (val.) $=8$ électrons par unité formularre $\mathrm{BN}$ ) sı l'on ne fait pas intervenur l'occupation des ES. Le bilan des transferts électronıques est alors :

$$
\begin{aligned}
n_{\mathrm{T}}(\mathrm{B})+n_{\mathrm{T}}\left(\mathrm{ES}_{\mathrm{B}}\right) & =2.71 \\
n_{\mathrm{T}}(\mathrm{N})+n_{\mathrm{T}}\left(\mathrm{ES}_{\mathrm{N}}\right) & =5.29 \\
n_{\mathrm{T}}(\mathrm{BN}) & =8.000=Q \text { (valence) } .
\end{aligned}
$$

Cecı amène à la formulation survante pour $\mathrm{BN}: \mathrm{B}^{+0,29} \mathrm{~N}^{-0,29}$ 
Tableau I. - BN-c: $a=7,002$ u.a., $\quad r\left(\mathrm{~B}, \mathrm{~N}, \mathrm{ES}_{\mathrm{B}}, \mathrm{ES}_{\mathrm{N}}\right)=1,725$ u.a. Partie gauche: charges partielles locales sur chaque espèce atomique. Partie droite : énergles propres (Ryd.) des fonctions sphériques de type Hankel des différents états. $E_{\mathrm{VAL}}=1,038 \mathrm{Ryd}(14,12 \mathrm{eV})$.

[c-B : $a=7,002$ u.a., $r\left(\mathrm{~B}, \mathrm{~N}, \mathrm{ES}_{\mathrm{B}}, \mathrm{ES}_{\mathrm{N}}\right)=1.725$ u.a. Left hand part : local partial charges on each atomic species. Right hand part : eigenenergies (Ryd.) of the spherical wave functions (Hankel) for the different states. $\left.E_{\mathrm{VAL}}=1.038 \mathrm{Ryd}(14.12 \mathrm{eV}).\right]$

\begin{tabular}{|c|c|c|c|c||r|c|c|}
\hline & $n_{\mathrm{s}}$ & $n_{\mathrm{p}}$ & $n_{\mathrm{d}}$ & $n_{\mathrm{T}}$ & $\begin{array}{c}\mathrm{E}(\mathrm{s}) \\
\text { Hankel }\end{array}$ & $\begin{array}{c}\mathrm{E}(\mathrm{p}) \\
\text { Hankel }\end{array}$ & $\begin{array}{c}\mathrm{E} \\
\text { Hankel }\end{array}$ \\
\hline $\mathrm{B}$ & 0,618 & 1,219 & 0,099 & 1,936 & $0,648 * *$ & 1,610 & 5,662 \\
\hline $\mathrm{N}$ & 1,368 & 3,237 & 0,032 & 4,637 & $-0,341 * *$ & $0,993 * *$ & 5,505 \\
\hline $\mathrm{es}(\mathrm{B})$ & 0,303 & 0,351 & 0,123 & 0,777 & 1,464 & 3,804 & 7,237 \\
\hline $\mathrm{es}(\mathrm{N})$ & 0,282 & 0,280 & 0,086 & 0,648 & 1,408 & 3,754 & 7,189 \\
\hline
\end{tabular}

Un tel transfert traduit une liaison $B \ldots N$ intermédiarre entre homopolaire et neutre plutôt qu'entre homopolaire et Ionique. Avec un transfert de charge global de $\delta=0,29 e^{-}$en faveur de $\mathrm{N}$, cette valeur est inférieure à celle obtenue par des calculs APW non-autocohérents : $\delta=0.35$ ma1s présente un sens opposé à celui annoncé par de tels calculs qu1 concluent à un transfert $\mathrm{N} \rightarrow \mathrm{B}[9]$.

Les énergies propres des fonctions d'ondes de type Hankel obtenues pour les différents états $\ell$ sont données à la partie droite du tableau $\mathrm{I}$. Il est intéressant de noter que pour les états $\mathrm{s}$ et $\mathrm{p}$ les énergies sont plus faibles pour $\mathrm{N}$ que pour $\mathrm{B}$, ceci est en accord avec le transfert de charges du second vers le premier. L'énergie de l'état $\mathrm{p}$ du bore est supérieure à celles des états $\mathrm{s}$ des deux ES indiquant sont caractère fortement antiliant. Les valeurs marquées d'étoiles dans le tableau I sont les énergies des états se trouvant en dessous du haut de la bande de valence : $E($ val $)=14,12 \mathrm{eV}$. En effet c'est cette valeur qui servira de repère - zéro - d'énergie, le « niveau de Fermi » se trouvant dans le gap. Les états dont les énergies sont impliquées dans la bande de valence sont indicatifs d'une hybridation pouvant s'apparenter au type $\mathrm{sp}^{3}$ avec un caractère p prédominant de $\mathrm{N}$ - les états p de $\mathrm{B}$ étant au-dessus de $E$ (val). Ce résultat attendu pour un semi-conducteur de structure tétraédrique pourrait être en relation avec la dureté de BN-c (9 Moh), voisine de celle du diamant (10 Moh) (cf. [1]). Enfin les états d pour toutes les espèces en présence sont trop éloignés énergétiquement de ceux proches des gaps et ne présentant pas d'intérêt physique.

4.3.2 Densités de charges dans les sphères atomiques. - Afin d'illustrer les distributions de charges, nous montrons à la figure 1 la distribution radiale dans chacune des sphères atomiques des densités $\rho_{\nu}(r)$ normalisées ( $\nu$ : indice de sphère). Ces tracés montrent plusieurs aspects.

- Dans la région de cœur (entre 0 et 0,5 u.a.), la densité de charges subit les fortes oscillations attendues pour cette régıon. De plus $\rho(r)_{\text {cœur }}(\mathrm{N})>\rho(r)_{\text {cœur }}(\mathrm{B})$ est un résultat en accord avec l'accumulation de charges « $\mathrm{S}$ » pour $\mathrm{N}$ (Tab. I). Notons que $\rho(r)_{\text {cœeur }}(\mathrm{ES})=0$, en accord avec l'absence d'états de cœur pour les sphères vides.

- Dans la régıon de semi-cœur et contrairement au bore, l'azote présente un nœud à $\cong 1,1$ u.a. correspondant à l'état $2 p$. 


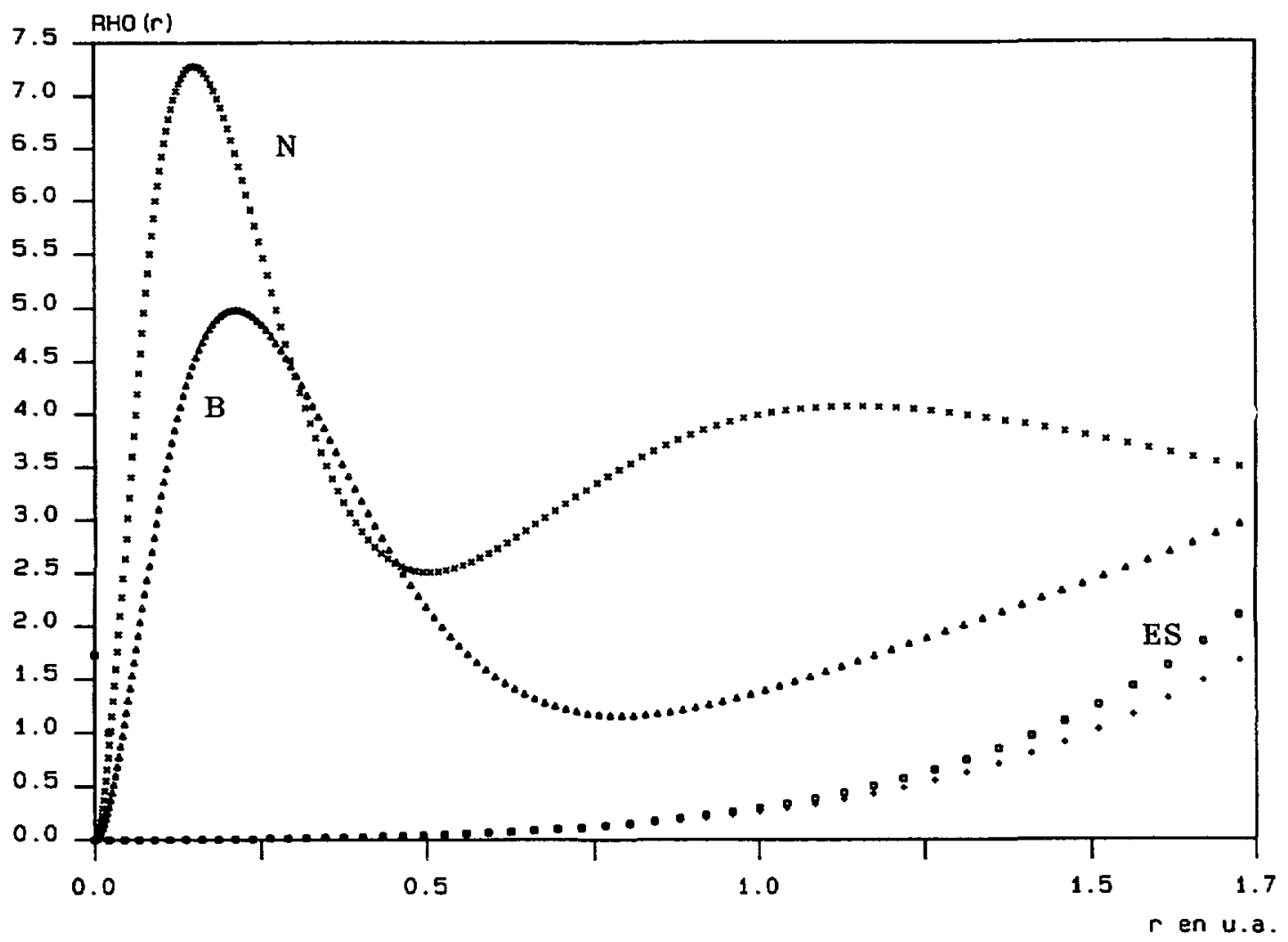

Fig. 1. - Distrıbution radiale des densités de charges normalısées à l'intérieur des sphères ASA pour BN-c.

[Normalised radial charge distribution in the ASA spheres for c-BN.]

- A la surface des sphères vides, $\rho(r)$ accuse une augmentation importante montrant une accumulation de charges. Ce résultat est en accord avec une région interstitielle essentiellement vide au point de vue densité électronique.

Notons enfin que cette évolution de $\rho(r)$ dépend bien entendu du choix des rayons des sphères et par suite est non unıque. Cependant les aspects essentiels n'en seront pas drastiquement modifiés pour un autre choix de rayons.

4.3.3 Densités d'états et structure de bandes. - La partie droite de la figure 2 montre les densités d'états (DOS) pour BN-c au paramètre $a_{\exp }$ obtenues pour 182 points k. Les énergies sont comptées par rapport au sommet de la bande de valence en $\Gamma_{15}^{\mathrm{v}}$ à $14,12 \mathrm{eV}$. Notons que la structure fine apparente des pics est due à un non filtrage numérıque des résultats mats ne doit pas influer sur le sens physique des DOS où seul l'aspect général est d'intérêt. Compte tenu des énergies données au tableau I, ces DOS sont attribuables de la manière suivante de bas en haut: DOS de $\mathrm{N}(\mathrm{s})$ majoritarres, mélange de DOS de $\mathrm{N}(\mathrm{p})$ et $\mathrm{B}$ (s) et, au-dessus du gap, DOS des ES suivies de celles de B(p). La présence des DOS relatives aux ES en bas de la bande de conductıon, aspect propre à la ASA et quı n'apparaît pas dans les travaux antérieurs, est à soulıgner. Il doit par exemple jouer un rôle sıgnificatif dans les propriétés électronıques lorsque les sites des ES seront occupés par des atomes interstitiels, ex. par dopage. 


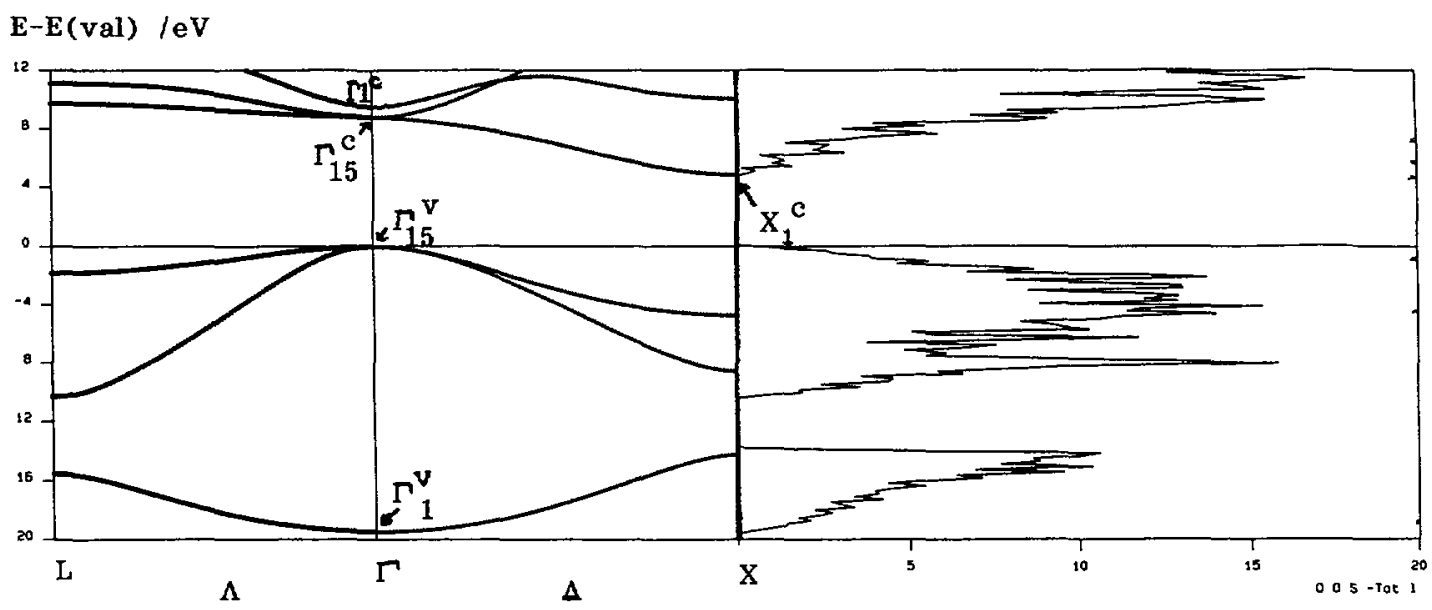

Fig. 2. - Représentation comparative des DOS et des bandes d'énergies pour BN-c. $E(v a l)=14,12 \mathrm{eV}$.

[Comparative representation of the DOS and of the band structure of c-BN. $E(\mathrm{val})=14.12 \mathrm{eV}$.]

L'analyse des seules DOS ne permet pas d'aboutır aux propriétés électroniques de BN-c notamment en ce qui concerne l'évaluation des gaps direct et indirect. La structure de bandes d'énergıe a été alors calculée dans la représentation irréductible de la première zone de Brillouin du réseau de Bravais du cubique à faces centrées. Conjointement aux DOS, la partie gauche de la figure 2 montre le tracé des bandes d'énergie suivant 2 directıons principales de la zone de Brillouin : $A$ et $\Delta$, solt $\mathrm{L}(1 / 2,1 / 2,1 / 2)-\Gamma(0,0,0)-\mathrm{X}(1 / 2,0,1 / 2)$ où $\Gamma$ désigne le centre de la zone. L'origine des énergies (axe y) est prise à $E(\mathrm{val})$, comme pour les DOS. L'aspect principal concerne le haut de la bande de valence qui se situe en $\Gamma$ alors que le bas de la bande de conduction se trouve en $\mathrm{X}$. Ceci est en accord avec le comportement de BN-c comme un semi-conducteur à gap indirect.

Energies de la largeur de la bande de valence et des gaps dans BN-c. - L'état de plus basse énergie est $\Gamma_{1}^{\mathrm{v}}$ (mono-dégénéré - états $\mathrm{s} ;$ 《 $\mathrm{v} »=$ valence), situé à $19,5 \mathrm{eV}$ en dessous de $\Gamma_{15}^{\mathrm{v}}$ (haut de la bande de valence). Le gap direct est entre $\Gamma_{15}^{\mathrm{v}}$ et $\Gamma_{15}^{\mathrm{c}}$ ( c $»=$ conduction) et vaut $8,8 \mathrm{eV}$, le gap indirect, entre $\Gamma_{15}^{\mathrm{v}}$ et $\mathrm{X}_{1}^{\mathrm{c}}$ vaut $4,9 \mathrm{eV}$. Ces valeurs sont confrontées au tableau II à celles calculées et expérımentales de la littérature. Pour ces dernières une certaine incertitude existe quant à leur amplitude ; les références [10] et [11] choisies dans le tableau II sont parmı les plus récentes.

Alors que les amplitudes de la largeur de la bande de valence et des gaps sont voisines pour les calculs basés sur la LDA : les nôtres aınsı que [2] à [5], ıl existe un certain désaccord entre ceux-cr et les calculs APW de Prasad et Dubey [9]. En outre, le meilleur accord avec les valeurs expérımentales pour les premıères - malgré un certain écart pour les valeurs des gaps - est à noter.

\section{Discussion.}

Prasad et Dubey [9] utılisent une méthode non-autocohérente de calcul (APW : augmented plane wave) basée sur des potentiels pour $\mathrm{B}$ et $\mathrm{N}$ tabulés par Herman et Skillman [12]. Notons que leurs calculs donnent de meilleures valeurs pour les troıs grandeurs consıgnées au tableau II lorsque BN est considéré avec une configuration ionique (valeurs données au tableau). En revanche les calculs basés sur la LDA sont effectués par des méthodes autocohérentes plus élaborées permettant, lors de la solution de l'équation d'onde dans un 
Tableau II. - Comparaison des énergies des gaps direct $\left(\Gamma_{15}^{\mathrm{v}}-\Gamma_{15}^{\mathrm{c}}\right)$ et indirect $\left(\Gamma_{15}^{\mathrm{v}}-\mathrm{X}_{\mathrm{l}}^{\mathrm{c}}\right)$ et de la largeur de la bande de valence $\left(\Gamma_{1}^{\mathrm{v}}-\Gamma_{15}^{\mathrm{v}}\right)$ pour BN-c.

[Comparison between the energies of the direct gap $\left(\Gamma_{15}^{\mathrm{v}}-\Gamma_{15}^{\mathrm{c}}\right)$, indirect gap $\left(\Gamma_{15}^{\mathrm{v}}-\mathrm{X}_{1}^{\mathrm{c}}\right)$ and of the valence band with $\left(\Gamma_{\mathrm{l}}^{\mathrm{V}}-\Gamma_{15}^{\mathrm{v}}\right)$ for c-BN.]

\begin{tabular}{|c|c|c|c|}
\hline $\begin{array}{c}\text { Energies } \\
\text { en eV }\end{array}$ & $\begin{array}{c}\text { Gap } \\
\text { direct }\end{array}$ & $\begin{array}{c}\text { Gap } \\
\text { indirect }\end{array}$ & $\begin{array}{c}\text { Bande de } \\
\text { valence }\end{array}$ \\
\hline Ref. [2] & 8,6 & 4,2 & 20,3 \\
\hline Ref. [3] & 8,8 & 4,4 & 20,1 \\
\hline Ref. [4] & 8,2 & 4,2 & 20,6 \\
\hline Ref. [5] & 8,7 & 5,2 & 21,1 \\
\hline Ref. [9] & 10,9 & 8,6 & 16,7 \\
\hline Ce trav. & 8,8 & 4,9 & 19,5 \\
\hline Experim. & 14,5 & $<6,0$ & $<22,0$ \\
{$[10]$} & {$[11]$} & {$[1]$} \\
\hline
\end{tabular}

potentiel effectıf d'un gaz d'électrons sans interaction, d'approximer correctement les effets d'échange et de corrélation. Nous limiterons notre discussion à ces calculs.

Il est intéressant de noter que l'accord pour la largeur de la bande de valence et le désaccord pour les gaps, plus important pour le gap direct que le gap indirect vont dans le sens d'états à densités électroniques de moins en moins grandes. La bande de valence est pleıne et sa largeur est bien reproduite par des calculs autocohérents basés sur la LDA; en revanche l'évaluation des gaps fait intervenır des états vides (bande de conduction) qui sont plus $\left(\mathrm{X}_{1}^{\mathrm{c}}\right)$ ou moins ( $\Gamma_{15}^{\mathrm{c}}$ ) rapprochés de la bande de valence. Or 1 a été établi par Godby et al. [8] que la LDA reste une bonne approxımation du potentiel d'échange et corrélation exact dans le cadre de la DFT. Par conséquent le désaccord - important pour le gap direct — provient du fait que ces calculs sont propres à l'état fondamental, 1 l n'est par suite pas a1sé d'établir des comparaisons directes avec des valeurs provenant de mesures d'états excités.

L'artéfact de la méthode ASW que représente l'introduction de sphères vides doit permettre en fait de simuler l'insertion d'atomes interstitiels (cf. Sect. 4.3) dans la structure blende des III-V [13]. Néanmoins, les mailles cristallınes sont alors plus importantes (mode primitıf, mailles multıples) et les moyens de calculs auss1. De telles études sont en cours sur BN-c et seront publiées dans un prochain papier.

\section{Bibliographie}

[1] Vel L., Demazeau G. and Etourneau J., Mat. Sci. Engın. B 10 (1991) 149 et références c1-incluses.

[2] Wentzcovitch R., Chang K. J. et Cohen M. L., Phys. Rev. B 34 (1986) 1071.

[3] Park K. T., Terakura K. and Hamada N., J. Phys. C : Solid State Phys. 20 (1987) 1241. 
[4] van Camp P. E., van Doren V. E. et Devereese J. T., Phys. Stat. Sol. (b) 146 (1988) 573.

[5] Xu Y.-N. et Ching W. Y., Phys. Rev. B 44 (1991) 7787.

[6] Willams A. R., Kübler J. et Gelatt C. D. Jr., Phys. Rev. B 19 (1979) 6094.

[7] Kohn W. et Sham L. J., Phys. Rev. 140 (1965) A1133.

[8] Godby R. W., Schlüter M. et Sham L. J., Phys. Rev. B 37 (1988) 10159.

[9] Prasad C. and Dubey J. D., Phys. Stat. Solidi. B 125 (1984) 629.

[10] Landolt-Börnstein New Series (1982): Physics of Group IV Elements and III-V Compounds. Group III, 17a, O. Madelung Ed. (Berlın: Springer Verlag).

[11] Chrenko R. M., Solid State Commun. 14 (1974) 511.

[12] Herman F. and Skillman S., Atomıc Structure Calculations (Prentice-Hall, Inc., Englewood Cliffs N.J., USA, 1963).

[13] Rompa H. W. A. M., Schuurmans M. F. H. et Willams F., Phys. Rev. Lett. 52 (1984) 675. 